\begin{tabular}{|c|c|c|}
\hline 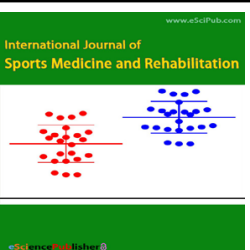 & $\begin{array}{l}\text { International Journal of Sports Medicine and Rehabilitation } \\
\qquad(\text { ISSN:2637-5044) }\end{array}$ & 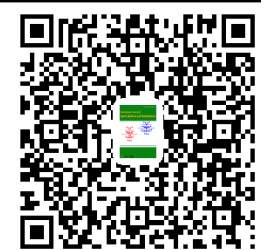 \\
\hline
\end{tabular}

\title{
The biceps brachii role in the stabilization of the cross punch
}

\section{Marco Prenassi ${ }^{1}$, Renato D. Donato ${ }^{2,3}$, Simona Mrakic-Sposta ${ }^{4 *}$, Lorenzo Rossi ${ }^{5}$, Luca Andreoletti ${ }^{3}$, Alessandra Vezzoli ${ }^{4}$, and Sara Marceglia ${ }^{6}$}

${ }^{1}$ UO Neurofisiopatologia, Fondazione IRRCS Ca'Granda Ospedale Maggiore Policlinico, Milan, Italy; ${ }^{2}$ Heracles Gymnasium SSD s.r.I. Fortitudo ETS, Milan, Italy; ${ }^{3}$ Dipartimento di Scienze Biomediche per la Salute,Università degli Studi di Milano, Milan, Italy; ${ }^{4}$ Institute of Clinical Physiology, National Research Council, IFC -CNR, Milan, Italy; ${ }^{5}$ Newronika s.r.l.; ${ }^{6}$ Dipartimento di Ingegneria e Architettura, Università degli Studi di Trieste, Trieste, Italy

\section{ABSTRACT}

Purpose: starting from an injury background, we assumed that the biceps brachii's activations could have an important role in Simona Mrakic-Sposta upper limbs injury. In this work we analyzed whether different Institute of Clinical Physiology, activations of the biceps brachii impact on the power transfer National Research Council, IFC of the punch and how boxers of different skill levels activate the CNR, Milan, Italy biceps brachii when deliver a punch according to their skill level and efficacy.

How to cite this article:

Methods: we enrolled, basing on official rankings, 23 skilled $(n=6)$ and unskilled boxers. Subjects were instructed to perform three cross punches directed to a fixed elastic target triggered by the coach whistling, and were monitored through a surface electromyography sensor (EMG) on the biceps brachii to estimate the muscular activation during the performance, and through an accelerometer placed inside the elastic impact target to estimate the impact energy. We analyzed the oscillatory content of the EMG signal in order to assess the muscular activation between skilled and unskilled boxers, and between weak and strong punches.

Results: both skilled and unskilled boxers threw strong, medium and weak strikes. Skilled boxers performed better than unskilled boxers ( $47 \%$ vs $25 \%$ in the "strong punch" category).The EMG analysis revealed a significant increase of lower and higher frequencies $(2-4 \mathrm{~Hz}$ and $15-17 \mathrm{~Hz}$ ) and a decrease on the

Marco Prenassi, Renato D. Donato, Simona Mrakic-Sposta, Lorenzo Rossi, Luca Andreoletti, Alessandra Vezzoli, and Sara Marceglia. The biceps brachii role in the stabilization of the cross punch. International Journal of Sports Medicine and Rehabilitation, 2020; $3: 13$

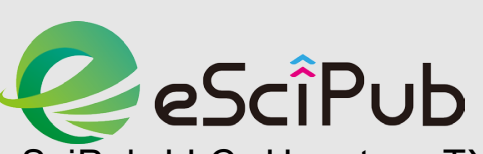
eSciPub LLC, Houston, TX USA. Website: https://escipub.com/ 
medium frequencies $(7-9 \mathrm{~Hz})$ in the skilled boxers compared to the unskilled boxers in strong punches. Weak punches had a similar activation patterns in the two groups.

Conclusions: our results support the hypothesis that skilled boxers adapt their activation pattern of the biceps to better stabilize the punch delivery (and thus increasing the transfer of force).

Keywords: Boxing; Biceps brachii; EMG analysis; Punch; Shoulder injury; Tendon detachment

\section{Introduction}

Boxing discipline is a sport characterized by many traumatic phenomena. Indeed, the fighters have to absorb the impact of the blows, to the head and the body, thrown by the rival but also, they have to absorb the impact of their own punches. Self-inflicted hand injuries could arise from these latter impacts.

Many studies investigate direct injuries to the hand and the head caused by the traumatic fist events. In particular, they focused on concussions, in short and long terms ${ }^{[1,2,3]}$ and also on metacarpal injuries $[4,5,6,7]$. Not less important, however, is the indirect trauma, caused by repetitive training sessions, to the muscles and tendons. The impact overload can direct the athletes to have tendinopathy, muscle tears, and, at worst, tendons detachment. Novice boxers have been shown to have fewer shoulder injuries than experienced boxers; this statistical evidence supports the idea that indirect trauma injuries, caused by functional overload, are no less important than direct trauma injuries. Shoulder injuries etiology by overload training can be identified in the defective movement of the blow. Incorrect movement patterns can stress the shoulder and set movement patterns characterized by scapular dyskinesia with an increase of extra-rotation ${ }^{[8]}$. Moreover, it can add an inaccurate control of scapular muscle and a bad activation of the serratus anterior that can create a

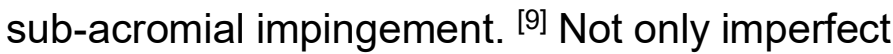
skills are dangerous for athletes' structures; a perfect shot still increases the overload of the impact. It is supposed that expert boxers can generate more impact force compared to novice boxers, and more impact force means an increased stress to the throwing arm.

These force impacts, generated by the punch when hits the target, stress the upper limb joints. Studies on these impact forces managed to demonstrate that, during the impact, the high activation of flexor and extensor muscles, recruited to close the hand, produce a shot with $55 \%$ more strength compared with a shot without clenched hand. The high muscle activation reduces injuries, metacarpal joint deformation, and increased power transfer to the target. ${ }^{[10]}$ The stabilizing muscle action generated by the impact on the target appears to be an involuntary kind, where the term "involuntary" is a not reflective event in line with the act's finality.

Exactly as the insights of Eugéne Minkowski in '57 when he described the body muscle compensation to keep the orthostatic position, which is in a continuous strain by perturbative force. [11] The body implements continuous compensation, without a real consciousness, intrinsically motivated, and intentioned, to reach the aim. As described by Minkowski E. (1957) and Lacquaniti \& Maioli (1989), the researches have shown that there are many anticipatory muscle activations, without the perception of consciousness, to stabilize posture and thus be able to initiate conscious action ${ }^{[11,12]}$.

An intentional impact, like a blow or a supportfoot under a jump, produces necessarily an involuntary muscle act to avoid unexpected results. 
This unconscious activation must be precisely tuned to the gesture to be performed. When there isn't tuning among anticipatory/compensator muscle activation and finality act, there is the probability of failure of the qualitative gesture or, in the worst, injuries.We can have a striking example in the fright and pain experienced during the loss of the last step when people go down the staircase without attention. The fails of anticipatory muscle activation obligate the body to compensate for the surprise of the impact force with the ground with the massive recruit of antigravitational chains and with a possibility of severe injuries to the back. Recent studies showed that the repeated impact force, arise from the foot support on the ground, generates electromyographic variances of the muscle activities to decrease soft tissue vibration ${ }^{[13]}$ and the overload tendon muscle. ${ }^{[14]}$ This evidence supports the idea that to the increment of the impact there is an increase of muscle recruit to reduce the overload of the joint and the tendon muscle structures.

The constant repetition of massive recruitment and hard force expression, generated from the repetitive specific workout, could be the principal cause of upper arm injuries, including long head brachii biceps muscle ${ }^{[15,16]}$. It is not infrequent that boxers report pain shoulder near the proximal attachment and, mostly in the heavyweight class, can suffer the laceration or complete detachment of the distal attachment. In the study conducted by Siewe et al, (2015) on boxing competition, the most frequent injuries, not including the injuries to the head, involve the upper limbs. [17] For this reason, it's important creating focused training programs, that can help the power increase of muscle groups stressed by impact force, could extend the boxer's sports career, and remove the risk of injuries ${ }^{[6,17]}$.

In boxing, the cross punch is a movement involving the hips, torso, and upper arm, in which the weight is transferred from the rear foot up to the knuckles of the hand ${ }^{[18]}$.

Nakano et al, (2014) ${ }^{[19]}$ suggests that the impulse of the cross punch to the face is more dependent on the final pre-impact momentum of the punching arm rather than the momentum of the other body segments, so its extension before the impact is vital in delivering a "strong" punch. In this complex action, the biceps brachii may be involved in dampening the extension velocity of the arm, limiting the impact force of the punch, or, after the impact; it may contribute to stiffening the arm and consequentially increasing the energy transfer. In this work, we also argue that this stiffening motion could be linked to the inflammation of the long head of the biceps brachii.

To better understand the role of the correct modulation of the biceps brachii activation in the cross punch stabilization, in this study we aim to: a) assess whether different activations of the biceps brachii impact on the power transfer of the punch; b) analyze how boxers of different skill levels activate the biceps brachii when deliver a punch according to their skill and efficacy. To do so, we neurophysiologically analyzed the response of the biceps brachii while boxers of different skill levels were executing a cross punch, by measuring the activation of this muscle with a bipolar superficial electromyogram (EMG) and an accelerometer inside an elastic target to assess the impact force between punches.

\section{Materials and Methods}

Twenty-three boxers were enrolled in this study and divided into two groups (skilled-unskilled) using the Italian Boxing Federation (FPI, Federazione Pugilistica Italiana), Association Internationale de Boxe Amateurs (AIBA) Open Boxing (AOB) score system [20]. We included in the skilled boxers with $>80$ scores. All subjects signed informed consent and were fully informed 
about the experimental procedure, according to the declaration of Helsinki.

After a baseline assessment to measure height, weight, and contracted biceps (bicepsC) circumference, boxers were asked to perform a Maximum Voluntary Contraction (MVC) task that was used as reference for subsequent analyses.
After that, they were instructed to perform three cross punches directed to a target upon hearing the coach whistle, using the regulated hand wraps and boxing gloves ${ }^{[21]}$. The elastic target was made by a wooden plate surrounded by an elastic rubber to absorb the impact (Figure 1).

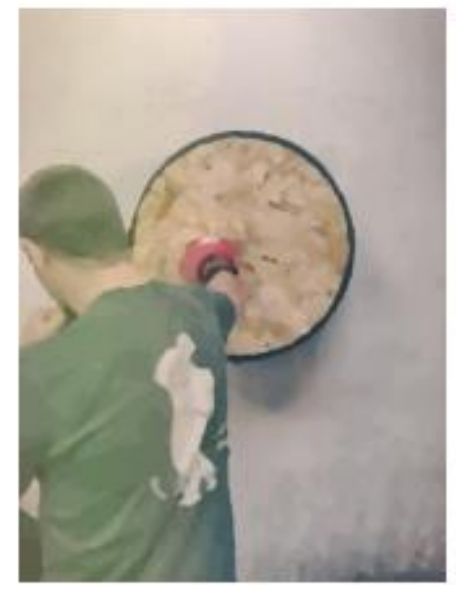

Figure 1. Experiment setup, boxer position and target.

At least 40 seconds elapsed between each starting signal (whistle). A 256 samples/s bipolar $E M G$, with one electrode placed at the origin and the other one at the insertion of the biceps brachii, was used to record muscle activity during the task. The reference electrode was applied to the lateral malleolus. A data-marker button was pushed to trigger the recording to the coach whistle. A three-axial accelerometer acquiring data at 100 samples/s (Shimmer Sensing IMU) was placed inside on the wooden plate, on its longitudinal axis of mass, in order to record the impact, as the energy transferred by the punch to the target. A visual analog scale of pain (VAS) was administered before and after the session, to assess the presence of pain and the area where the pain was predominant.

The punch impact was estimated through the accelerometer data placed within the target and calculated within a 10-sec window centered on the maximum absolute peak of acceleration. We estimated the relative energy transferred by the punch with this formula:

$$
r E_{k}=\frac{E_{k}}{m}=\sum_{i=0}^{10 \cdot F s}\left|a_{i}\right|^{2}\left[\frac{j}{k g}\right]
$$

$i=0$ where $r E_{k}$ is the signal of relative energy, $E_{k}$ is the kinetic energy, $m$ the mass of the target (the target is the same for every boxer), $a_{i}$ : instant acceleration detected by the sensor and $F s$ is the sampling frequency (100 samples/s). We omitted the energy absorbed by plastic deformations of the target, since it was regarded as a minor component during the trials (the target accelerometric response was tested on the floor, dropping a ball weight of $5 \mathrm{~kg}$ at 1 meter before and after the experiment, the results were comparable within $2 \%$ of the total power). 
The biceps brachii activation was estimated through the EMG signal recorded during the execution of the cross punch. More specifically, the full rectified EMG signal was normalized by its MVC. We selected the cross-punch EMG window using the data marker trigger as initial point (corresponding to the whistle). The end point was selected as the instant in which the signal, smoothed with an average mean filter by 256 samples, crossed its standard deviation (calculated on the entire recording). To assess the activation of the biceps brachii, we analyzed the oscillatory content of the EMG signal in each cross- punch window. This is represented by the power spectrum of the EMG signal calculated using the Welch method ${ }^{[22]}$ which shows the predominant frequencies in the EMG signal. We considered the envelope of the EMG as smoothing the rectified signal with a low pass filter ${ }^{[23]}$ with a time constant of $4 \mathrm{~ms}$.

Punches were sorted according to the skill level of the boxer (skilled and unskilled) and by their relative kinetic energy, to categorize "strong", "weak", and "medium" punches, according to the following 2 thresholds (Figure 2):

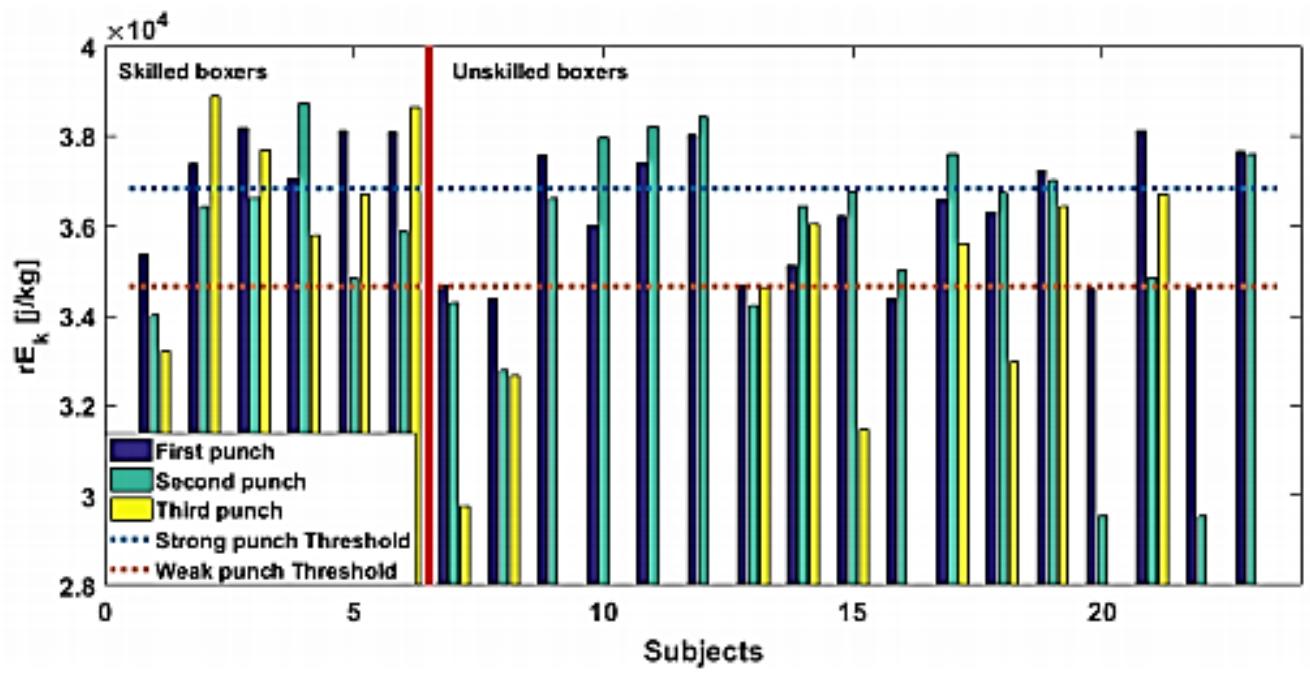

Figure 2. Relative punch energy per subjects, grouped by skilled/unskilled boxers and with strong, medium, weak punch thresholds.

$$
\left\{\begin{array}{l}
r E_{k i} \geq 0.33 \cdot s t d\left(r E_{k}\right) \\
0.33 \cdot \operatorname{std}\left(r E_{k}\right) \geq r E_{k i}>-0.33 \cdot \operatorname{std}\left(r E_{k}\right) \\
r E_{k i}<-0.33 \cdot \operatorname{std}\left(r E_{k}\right)
\end{array}\right.
$$

strong punch medium punch weak punch

Where std is the standard deviation.

Table 1

NUMBER OF PUNCHES BY CATEGORY

\begin{tabular}{lcccc} 
& \multicolumn{3}{c}{ NUMBER OF PUNCHES BY CATEGORY } \\
\cline { 2 - 5 } & Strong & Medium & Weak & $\begin{array}{c}\text { Total } \\
\text { punches } \\
\text { by skill }\end{array}$ \\
\hline Skilled & 8 & 7 & 2 & 17 \\
& $47 \%$ & $41 \%$ & $12 \%$ & $100 \%$ \\
Unskilled & 9 & 15 & 12 & 36 \\
& $25 \%$ & $42 \%$ & $33 \%$ & $100 \%$ \\
$\begin{array}{l}\text { Total } \\
\text { punches } \\
\text { by rEk }\end{array}$ & 17 & 22 & 14 & 53 \\
\hline
\end{tabular}


Table 2

\begin{tabular}{ccc}
\multicolumn{3}{c}{ CORRELATION WITH RELATIVE SIGNAL ENERGY } \\
\hline Measure & $\begin{array}{c}\text { Mean } \\
r E k^{*}\end{array}$ & \\
\hline Height & 0.81 & $\mathrm{p}<0.01$ \\
Weight & 0.78 & $\mathrm{p}<0.01$ \\
$\begin{array}{c}\text { Contracted } \\
\text { biceps } \\
\text { circumference }\end{array}$ & 0.51 & $\mathrm{p}<0.075$
\end{tabular}

* Mean of the first two punches for every boxer

Table 1 shows the number of punches associated for each category. We therefore compared the oscillatory activities of the EMG signal in skilled and unskilled boxers and in strong, medium, and weak punches.

Then, to rule out the presence of impact artefacts, affecting the estimation of biceps brachii activation, we verified the relationship between the signal recorded from the muscle and that recorded from the target. More specifically, we performed a coherence analysis between the power spectra of the accelerometric raw signal and the EMG envelope on the whole cross punches.

Finally, we compared the pain perception in skilled and unskilled boxers measured through the VAS, by applying a Wilcoxon-Mann-Whitney test $(p<0.05)$.
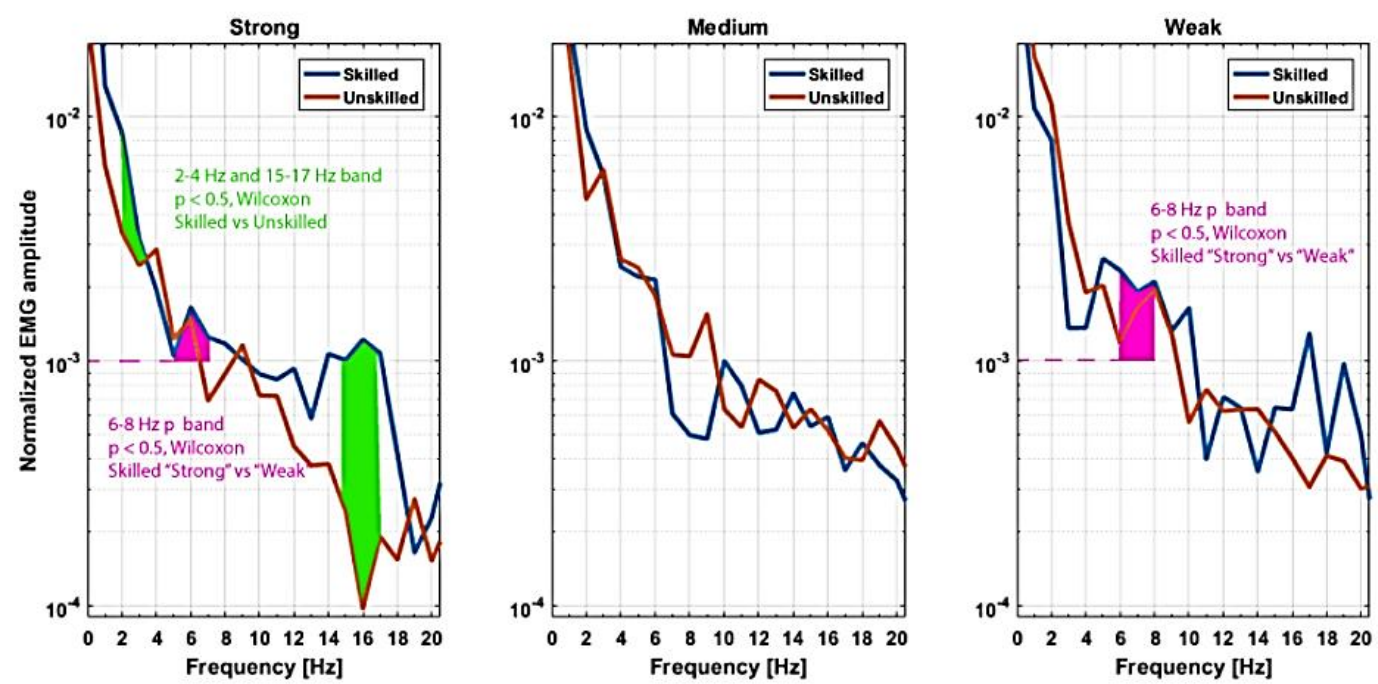

Figure 3. Mean MVC EMG power density spectrum between the categories ("Strong" punches, "Medium" punches and "Weak" punches) and the skill level (Skilled and Unskilled). The magenta and green areas are differences of statistical significance: $p<0.05$, Wilcoxon-Mann-Whitney test.

\section{Results}

Six boxers were enrolled in the skilled group (AOB score mean 140.4, standard deviation: $55.6)$, and 14 in the unskilled group (AOB score mean 11.5, standard deviation: 19.5). Three were discarded due to data loss. Of the 60 punches 7 were discarded due to a sensor malfunctioning. In total, we analyzed 53 punches, 17 for the skilled group and 36 for the unskilled group. Table 2 shows that the circumference of the biceps (BicepsC) is not correlated with the strength of the punch (mean $r E_{k}$ ) for each boxer. 
Also, we found 14 weak punches (of which 2 belonging to skilled boxers), 22 medium punches (of which 7 belonging to skilled boxers), 17 strong punches (of which 8 belonging to skilled boxers). Table 1 and Figure 2 show that, even if the skilled group tends to strike with superior force, not all motions are stronger than the unskilled counterpart.

We therefore analyzed the activation of the biceps brachii according to the skill level and to the strength of the punch (Figure 3).

We found that in the "strong punch" category, there is a higher EMG activation in the $2-4 \mathrm{~Hz}$ and in the $15-17 \mathrm{~Hz}$ oscillatory ranges in skilled boxers compared to unskilled boxers. Conversely, the "weak punch" group is characterized by an oscillation at $6-8 \mathrm{~Hz}$, both in skilled and unskilled boxers. Notably, this peak is also present in the "strong punch" in unskilled boxers, but not in skilled boxers ( $p<0.05$, Wilcoxon).

In the "medium punch" category, we observed a difference in biceps brachii activation between the skilled and unskilled group in low and high frequencies $(2-4 \mathrm{~Hz}$ and $15-17 \mathrm{~Hz})$, similar to that of strong punches, even if not statistically significant. Then, the results of the correlation analysis aimed to rule out the presence of impact artifacts in signals, showed an high coherence (mean magnitude squared coherence estimate $>$ $80 \%$ ) at $1 \mathrm{~Hz}, 5 \mathrm{~Hz}, 9 \mathrm{~Hz}, 14 \mathrm{~Hz}$ and $19 \mathrm{~Hz}$, suggesting possible artefacts, and a low coherence (mean magnitude squared coherence estimate $<42 \%$ ) in $2 \mathrm{~Hz}, 7 \mathrm{~Hz}, 14-16 \mathrm{~Hz}$ bands, thus confirming the electrophysiological differences measured through the EMG.Three skilled boxers reported a slight increase of pain in the punching hand (mean difference +2.14 ), while only one unskilled boxer reported a slight increase (mean difference +0.714 ), with a significant difference between the two groups $(p<0.025)$. No pain increase was reported in other part of the body by all the boxers.

\section{Discussion}

In this preliminary study, we showed that the contribution of the biceps brachii to the cross punch differs between skilled and unskilled boxers, especially in strong punches, thus supporting the hypothesis that a correct modulation of the activation of this muscle is crucial for improving the punch efficacy.

Our results show a notable increase in the skilled group on the low frequencies $(2-4 \mathrm{~Hz})$ and the high frequencies $(15-17 \mathrm{~Hz})$ in strong punches but not in weak punches, in which the two groups show a similar activation pattern (the EMG 6-8 $\mathrm{Hz}$ peak is present in both groups). In the "strong punch" category, this peak at 6-8 $\mathrm{Hz}$ is present only in the unskilled group. The activation of biceps brachii is suggested to change in the medium group, where the central frequencies tend to decrease in the skilled group compared to the unskilled group. These different patterns could also be linked to the inflammation of the long head of the biceps brachii, a common occurrence in boxing.

This inflammation is often associated to a limited articular mobility of the scapula and to a strong intra rotation of the shoulder caused by the specificity of the athletic gesture. The etiology of this inflammation is thought to be associated to an indirect phenomenon caused by a non-efficient shoulder joint biomechanics. Our work suggests, instead, that the inflammation can be caused by the incorrect recruitment of the biceps brachii during strong punches. The performance model of the punch needs to consider the different activation to reduce inflammations, elongations, strains or muscle/tendons tears.

There are however some technical limitations to be considered. First, the recording hardware (EMG device with 256 samples/s sampling with only one channel) could not be synchronized 
with the accelerometer device thus preventing us from performing more in-depth analyses of the correlation between muscle activity and impact. Second, EMG electrodes were placed further away from each other, enabling possible crosstalk with the triceps brachii; this was done in order to not impair the punch movement of the athletes. In the future, these limitations should be overcome in order to conduct studies aimed to characterize the EMG signals using a finer timescale synchronized with the impact of the punch, and to analyze the precise activation of both biceps brachii and triceps brachii before and after the hand contact with the target.

In addition, the changes in the activation pattern of the biceps brachii observed in the skilled group and the variation of the athletic gesture supports a philosophical thesis about learning through selfimitation ${ }^{[24]}$. The skilled boxer, trying to find the most effective athletic gesture, is capable of developing his/her punching method thanks to an explorative motion that trough "trials and errors" have helped him/her to identify the best technique for his/her corporeality. This variability have an impact in the teaching technique which shouldn't be strictly analytical but rather give room for a phase of "learning by error" to develop new boxing skills, with the final objective of finding new methods that fit the psychophysical characteristics of the boxer; that is accomplished with feedbacks of effectiveness and gratification. The analysis of muscular activity, and specifically of the biceps brachii, could guide the trainer in understanding whether the athletic gesture developed by the boxer is safe enough in terms of possible inflammations or other adverse effects.

Practical applications: Even if preliminary, this study suggests that the frequent inflammation phenomena, or in the worst case, the detachment of the distal insertion of the biceps brachii, could be caused by the lack of conditioning and strain-adaptation. This maximal recruitment of the muscular district, caused by the impact forces, could be a reflex contraction to safeguard the shoulder or humeroradial joint. A correct training must therefore take into consideration this dampening action.

In the light of this new performance model hypothesis, to counterbalance the strong reflex action of the biceps brachii and to avoid injuries, new complementary exercises could be added to the flexor chain of the daily routine of unskilled boxers, focusing on the power modulation of the shots on different boxing bags (light/medium/heavy) to gradually adapt both tendons to the impact forces.

These complementary exercises could be either general or specific. Examples of general exercises are:

1. Slow curl with elastic tapes with antalgic function;

2. Curl with dumbbells with emphasis on eccentric phase to strengthen the frontal biceps;

3. Pull ups to strengthen the dorsal muscles and the biceps brachii;

4. Hand grip strength exercises; and of specific exercises:

1. Single punches to the heavy boxing bag searching for the adaptation on multiple impact angle;

2. Power modulation of the hits with different boxing bags (light/medium/heavy) to adapt gradually the tendon to the impact forces.

This strategy requires further studies aimed to collect the long-term results of the proposed exercises in relation to the muscle inflammation, in order to establish its efficacy in reducing inflammation and injuries in unskilled boxers. In this brief exercise, no pain increase was reported in that location, the predominant pain was located on the hand and relevant only in the skilled 
boxer's category.

\section{Conclusions}

In a more general framework, our study showed that a quantitative and objective analysis of the activation patterns of specific muscles is able to discriminate between skilled and unskilled sportsman. Therefore, taking the skilled boxers as reference, is possible to provide suggestions on the optimization of training activities in order to boost the efficacy of the athletic gestures.

Acknowledgement: The authors are grateful to all the athletes that participated in the experimentation.

\section{References}

[1] Förstl H, Haass C, Hemmer B, Meyer B, Halle M. Boxing-acute complications and late sequelae: from concussion to dementia. Dtsch Arztebl Int. 2010 Nov;107(47):835-9. doi: 10.3238/arztebl.2010.0835. Epub 2010 Nov 26. Review. PubMed PMID: 21173899; PubMed Central PMCID: PMC3003467

[2] McCrory P, Zazryn T, Cameron P. The evidence for chronic traumatic encephalopathy in boxing. Sports Med. 2007;37(6):467-76. Review. PubMed PMID:17503873

[3] Blennow K, Popa C, Rasulzada A, Minthon L, Wallin $A$, Zetterberg $H$. There is a strong evidence that professional boxing results in chronic brain damage. The more head punches during a boxer's career, the bigger is the risk. Lakartidningen. 2005 Sep 5-11;102(36):2468-70, 2472-

5. Review. Swedish. PubMed PMID: 16196432.

[4] Ribeiro LM, Botton MA. Isolated Trapezoid Fracture in a Boxer. Am J Case Rep.2019 Jun 5; 20:790-793. doi: 10.12659/AJCR.915757. Review. PubMed PMID: 31164626; PubMed Central PMCID: PMC6561143.

[5] Melone CP Jr, Polatsch DB, Beldner S. Disabling hand injuries in boxing: boxer's knuckle and traumatic carpal boss. Clin Sports Med. 2009 Oct;28(4):609-21, vii. doi: 10.1016/j.csm.2009.06.004. Review. PubMed PMID:19819405.

[6] Noble C. Hand injuries in boxing. Am J Sports Med. 1987 Jul-Aug;15(4):342-6. PubMed PMID: 3661815.

[7] Hanna WA, Laier P. Hand injuries from combat sports. Unfallchirurg. 2015 Jun;118(6):507-14. doi: 10.1007/s00113-015-0014-y. Review. German. PubMed PMID: 26013389.

[8] Lenetsky, S., Brughelli, M., And Harris, N.K. 2015. Shoulder function and scapular position in boxers. Phys. Ther. Sport Off. J. Assoc. Chart. Physiother. Sports Med. 16, 355-360

[9] Kaur, N., Bhanot, K., Brody, L.T., Bridges, J., Berry, D.C., and Ode, J.J. 2014). Effects of lower extremity and trunk muscles recruitment on serratus anterior muscle activation in healthy male adults. Int. J. Sports Phys. Ther. 9, 924-937.

[10] Horns, J., Jung, R., And Carrier, D.R. 2015. In vitro strain in human metacarpal bones during striking: testing the pugilism hypothesis of hominin hand evolution. J. Exp. Biol. 218, 3215-3221.

[11] Minkowski, E., forword on "Attitudes et mouvements" di F.J.J Buytendijk, Bruges, Descléè de Brouwer, 1957, p.5

[12] Lacquaniti F, Maioli C. The role of preparation in tuning anticipatory and reflex responses during catching. J Neurosci. 1989 Jan;9(1):134-48

[13] Nigg Bm, Wakeling Jm, Impact forces and muscle tuning: a new paradigm. Exerc Sport Sci Rev. 2001;29(1):37-41

[14] Nigg Bm. The role of impact forces and foot pronation: a new paradigm. Clin J Sport Med. 2001 Jan;11(1):2-9. Review

[15] Lemme Nj, Ready L, Faria M, Defroda Sf, Gil Ja, Owens Bd. Epidemiology of boxing-related upper extremity injuries in the United States. Phys Sportsmed. 2018 Nov;46(4):503-508.

[16] Jordan Bd, Voy Ro, Stone J. Amateur Boxing Injuries at the US Olympic Training Center, Phys Sportsmed. 1990 Feb;18(2):80-90 
[17] Siewe J, Rudat J, Zarghooni K, Sobottke R, Eysel P, Herren C, Knöll P, Illgner U, Michael J. Injuries in competitive boxing. A prospective study. Int J SportsMed. 2015 Mar;36(3):249-53

[18] Tong-lam R, Rachanavy P, Lawsirirat C. Kinematic and kinetic analysis of throwing a straight punch: the role of trunk rotation in delivering a powerful straight punch. J Phys Educ Sport. 2017;17(4):2538-2543.

[19] Nakano G, lino Y, Imura A, Kojima T. Transfer of momentum from different arm segments to a light movable target during a straight punch thrown by expert boxers. J Sports Sci. 2014;32(6):517-523.

[20] Federazione Pugilistica Italiana. Regolamento A.O.B.http://www.fpi.it/fpi/statuto-e-regolamenti. html. Published July 2017.

[21] A.O.B. Open Boxin. A.I.B.A. Regulations, Glove Specifications and Guidelines, Appendix E. 2017.

[22] Welch P. The use of fast Fourier transforms for the estimation of power spectra: a method based on time averaging over short, modified periodograms. IEEE Trans Audio Electroacoustics. 1967;15(2):70-73.

[23] Merletti R, Di Torino P. Standards for reporting EMG data. J Electromyogr Kinesiol. 1999;9(1):34.

[24] Mormino G. Per Una Teoria Dell'imitazione. Raffaello Cortina Editore; 2016. Raffaello Cortina Editore; 2016.

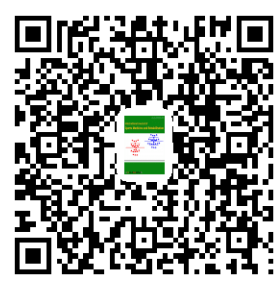

